\title{
Estimation of Domain Means from Business Surveys in the Presence of Stratum Jumpers and Nonresponse
}

\author{
Mengxuan $X u^{1}$, Victoria Landsman ${ }^{1}$, and Barry I. Graubard ${ }^{2}$
}

\begin{abstract}
Misclassified frame records (also called stratum jumpers) and low response rates are characteristic for business surveys. In the context of estimation of the domain parameters, jumpers may contribute to extreme variation in sample weights and skewed sampling distributions of the estimators, especially for domains with a small number of observations. There is limited literature about the extent to which these problems may affect the performance of the ratio estimators with nonresponse-adjusted weights. To address this gap, we designed a simulation study to explore the properties of the Horvitz-Thompson type ratio estimators, with and without smoothing of the weights, under different scenarios. The ratio estimator with propensity-adjusted weights showed satisfactory performance in all scenarios with a high response rate. For scenarios with a low response rate, the performance of this estimator improved with an increase in the proportion of jumpers in the domain. The smoothed estimators that we studied performed well in scenarios with non-informative weights, but can become markedly biased when the weights are informative, irrespective of response rate. We also studied the performance of the 'doubled half' bootstrap method for variance estimation. We illustrated an application of the methods in a real business survey.
\end{abstract}

Key words: Doubled half bootstrap; propensity-adjusted weights; weight smoothing.

\section{Introduction}

Firm size plays an instrumental role in different stages of business surveys. The information about a firm's size (usually in the form of employment or annual sales), geographic area and business sector obtained from administrative data are often used to define sampling strata at the design stage of the survey. Additionally, firm size is believed to be related to the probability to participate in the survey. Since business surveys may be prone to low response rates (Cook et al. 2009), the information about firm size is useful for nonresponse adjustments of the sample weights. Firm size was also suggested to be an important proxy variable to a firm's occupational health and safety performance (Nordlöf et al. 2015). For this reason, there is a lot of interest to evaluate and compare various performance metrics between firms of different sizes. Frequently, the target parameter is expressed as the domain mean where the domains are defined by the levels of firm size,

\footnotetext{
${ }^{1}$ Institute for Work and Health, 400 University Avenue, Suite 1800, Toronto, Ontario M5G 1S5, Canada. Emails: mengxuan.xu@mail.utoronto.ca and vlandsman@iwh.on.ca

${ }^{2}$ National Cancer Institute, Division of Cancer Epidemiology and Genetics, Bethesda, MD 20852, U.S.A. Email: graubard@mail.nih.gov

Acknowledgments: The authors thank Drs. Ben Amick III, Sheilah Hogg-Johnson, and Ms. Colette Severin for providing the OLIP data and the inspiration for the jumpers problem, which motivated this study. The authors also express their gratitude to the Associate Editor and three anonymous referees whose valuable comments help to considerably improve the quality of this manuscript.
} 
usually collected at the time of survey. The information about firm size obtained from administrative data that is typically used for defining the sampling strata and nonresponse adjustments, may contradict the information obtained from survey participants. Such situations may occur, for instance, when a small firm expanded and became a large firm in the time period between the survey design and data collection. Firms for which the two sources of information do not agree have been referred to in the literature as 'misclassified frame records' or 'stratum jumpers' (MacNeil and Pursey 2002, Beaumont and Rivest 2009). This article aims to address challenges in estimating the domain means of organizational performance measures from business surveys with misclassified frame records and low response rates.

Firms are usually sampled with sampling fractions proportional to their size. Therefore, jumpers that were considered as small firms at the design stage would typically have very large sample weights. In contrast, the correctly classified large firms are frequently sampled with certainty or with probability close to 1 , and, therefore, have weights close to or equal to 1 . When the domains of interest are defined by the levels of the firm size variable collected at the time of the survey, the presence of just a few jumpers in the domain of large firms may cause a dramatic increase in the coefficient of variation (CV) of the sample weights in the domain. Moreover, if the probability of response is proportional to firm size, nonresponse adjustments applied to the sample weights may further increase the $\mathrm{CV}$ of the weights in the domain.

Highly variable sample weights are known to lead to inefficient weighted estimators for means and totals (Korn and Graubard 1999, 172-173). Also, design-based variance estimators have been shown to underestimate the variance of the weighted estimators with highly variable weights in case-control designs used in epidemiological studies (Li et al. 2011; Landsman and Graubard 2013). In addition, one might expect a skewed sampling distribution of the ratio weighted estimators for domains with a small number of observations (Lee 1995). All of these factors combined are expected to slow the rate of convergence of consistent design-based variance estimators and negatively affect the coverage probabilities of the confidence intervals (CIs) around the parameters of interest.

The winsorization method is often used to treat influential values of outliers in survey data (Chen et al. 2017). This method requires finding a threshold, above which values of the variable of interest or the sampling weights are 'trimmed' down to the threshold to reduce their influence. Usually, the value of the threshold is selected to minimize the design mean square error of the estimator with the winsorized weights, although other methods have been proposed more recently (Favre-Martinoz et al. 2015). The winsorization method is less appealing in business surveys with multiple variables of interest, because different cutoff values imply different winsorized weights for each variable. For this reason, we did not pursue winsorization in this study. Weight smoothing is another approach that has been proposed to address the problem of highly variable weights (e.g., Pfeffermann and Sverchkov 1999; Beaumont 2008). Beaumont and Rivest (2009) devised two weight smoothing procedures to address the problem of the influential weights of the jumpers for estimating a population total from a stratified random sample with known (fixed) selection probabilities. One of the two procedures is a special case of the model-based approach proposed in the work of Beaumont (2008), and the second procedure can be viewed as a mixture of winsorization and smoothing approaches. The authors further showed, using a 
real data example, that the Horvitz-Thompson $(\mathrm{H}-\mathrm{T})$ estimator for the total with smoothed weights may be superior to the standard H-T estimator with the original (base) weights.

To the best of our knowledge, the performance of ratio estimators with nonresponseadjusted weights, with and without smoothing of the weights, has not been studied previously in the context of business surveys with misclassified frame records. Designbased variance estimation in this context can also be challenging, especially when using smoothed weights. Beaumont and Rivest (2009) used a Rao-Wu rescaled bootstrap estimator (Rao and Wu 1988; Rao et al. 1992) with smoothed weights, but did not consider nonresponse.

In this article we describe the exploratory work designed to address these gaps in the analysis of business surveys. The rest of the article is organized as follows. In Section 2, we formalize the problem and define a target parameter for the case of full response. For extension beyond this case, nonresponse adjustments are briefly outlined in Section 3. In Section 4, we describe the two weight smoothing methods proposed by Beaumont and Rivest (2009) that we adapted to the estimation of domain means. Section 5 describes the variance estimation using the 'doubled half' bootstrap method (Antal and Tille 2014). In Section 6, we illustrate the application of the methods to real data from an organizational performance survey. A detailed description of the simulation study and our main findings are summarized in Section 7. We conclude with a discussion about our findings in Section 8 .

\section{Problem Statement}

Suppose that $U$ is a finite population (of size $N$ ) of all firms in a given jurisdiction, and $Z_{\text {surv }}$ is a firm size variable with $D$ categories defined at the time of the survey (hence, the subscript surv). Thereby, $Z_{\text {surv }}$ defines the partition of $U$ into $D$ domains. The domain means $\mu_{d}(d=1, \ldots, D)$ of a study variable $y$ are the target parameters. To estimate $\mu_{d}$, a stratified random sample $\tilde{S}$ (of size $\tilde{n}$ ) with $H>1$ strata is usually drawn from $U$. In business surveys, the strata are typically defined by the categories of firm size and other relevant variables (e.g., geographic region and business sector).

The values of the variable $Z_{\text {surv }}$ are usually unobserved for the firms in the finite population and will only be obtained for the responding firms. The proxy variable for the firm size, $Z_{\text {des }}$, is usually derived from the available information (e.g., administrative data) at the design stage (hence, the subscript des) and this is the firm size variable that is used to form the strata. Throughout this article, $Z_{\text {des }}$ has two values: small and large. As a result, multiple strata of small and large firms are created by all possible combinations of the two categories of $Z_{\text {des }}$ and the categories of additional variables used to defined the strata. For simplicity of presentation, in this section and in Section 4 we assume that $Z_{\text {surv }}$ also has two values, small and large, defined in the same way as the two levels of $Z_{\text {des }}$. In Sections 6 and 7, we consider the case where $Z_{\text {surv }}$ has five different values.

The two categories of $Z_{\text {surv }}$ define the partition of $\tilde{S}$ into two mutually exclusive and collectively exhaustive domains: $\tilde{S}_{\text {small }}-$ the domain of small firms and $\tilde{S}_{\text {large }}-$ the domain of large firms. Assuming full response, $\mu_{d}(d \in\{$ small, large $\})$ can be estimated from the corresponding sample domain $\tilde{S}_{d}$ using a standard Horvitz-Thompson type ratio estimator 


$$
\hat{\mu}_{d}=\sum_{i \in \tilde{S}_{d}} b_{i} y_{i} / \sum_{i \in \tilde{S}_{d}} b_{i}
$$

where $b_{i}$ is a base sampling weight of firm $i$ determined by the stratified design.

Discrepancies between the values of $Z_{\text {des }}$ and $Z_{\text {surv }}$ might occur, especially if the information on the frame, used to define $Z_{\text {des }}$, was outdated (e.g., a large gap in time between the design and implementation of the survey). In this article, we assume that no firms changed their size from large to small, and focus our attention on the situation when firms changed their size from small to large, as this situation is more challenging for practitioners. Under this assumption, the sample domain of large firms can be written as $\tilde{S}_{\text {large }}=\tilde{S}_{J} \cup \tilde{S}_{L}$, where

$$
\tilde{S}_{J}=\left\{i \in \tilde{S} \mid Z_{\text {des }, i}=\operatorname{smal1}, Z_{\text {surv }, i}=\text { large }\right\}
$$

and

$$
\tilde{S}_{L}=\left\{i \in \tilde{S} \mid Z_{\text {des }, i}=\text { large, } Z_{\text {surv }, i}=\text { large }\right\}
$$

Firms in $\tilde{S}_{J}$ have been referred to in the literature as 'misclassified frame records', or 'stratum jumpers'.

Probability proportional to size (PPS) sampling, often used in stratified designs, assigns small sampling fractions, or, equivalently, large $b_{i}$, to small firms, including the firms in $\tilde{S}_{J}$. Firms in $\tilde{S}_{L}$, on the other hand, are usually assigned sampling fractions with values close to or equal to 1 , or, equivalently, values of $b_{i}$ which are close to or equal to 1 . As a result, the weights of the firms in $\tilde{S}_{J}$ can be considerably higher than the weights of the firms in $\tilde{S}_{L}$, causing a dramatic increase in the CV of the weights in $\tilde{S}_{\text {large. This is }}$ problematic, as the domain mean estimator (1) with highly variable weights may lead to estimates with large variances if the correlation between the weights and the variables of interest is weak (Rao 1966).

\section{Nonresponse Adjustments}

In practice, survey data is available for only a subset of respondents $S$ rather than for the entire selected sample $\tilde{S}$. In such cases, adjustments to the base weights are used to reduce the nonresponse bias. The main idea behind various adjustment procedures proposed in the literature is to estimate a firm's response probability, $p_{i}$, using all of the available data for the firms in $\tilde{S}$. The nonresponse-adjusted weights are defined as $w_{i}=b_{i} \hat{p}_{i}^{-1}$, where $\hat{p}_{i}$ is the estimated response probability of firm $i$, and may be further calibrated to match known population totals (Haziza and Lesage 2016). For simplicity, we do not consider calibration in this article and define the propensity-adjusted weights $w_{i}$ as final sample weights. Then, the population domain mean $\mu_{d}$ can be estimated from a sample of respondents $S_{d}$ using a ratio estimator with nonresponse-adjusted weights $w_{i}$

$$
\hat{\mu}_{d}=\sum_{i \in S_{d}} w_{i} y_{i} / \sum_{i \in S_{d}} w_{i}
$$

Different procedures can be applied to estimate $p_{i}$, depending on data availability and research goals. If the only data available for the firms in $\tilde{S}$ is the data that was used to form the sampling strata, the response probability can be estimated by $\hat{p}_{i h}=n_{h} / \tilde{n}_{h}$, where $n_{h}$ is 
the number of respondents and $\tilde{n}_{h}$ is the sample size of stratum $h(h=1, \ldots, H)$ in $\tilde{S}$. This method has been referred to as the 'cell adjustment' method (Little and Vartivarian 2003, Valliant et al. 2013) and it assumes that nonresponse is 'completely at random' within each stratum. We denote the 'cell-adjusted' weights by $w_{\text {str }}$ throughout the article.

If additional information is available for the firms in the selected sample $\tilde{S}$ (e.g., by linking $\tilde{S}$ to administrative data), the response probabilities can be estimated by fitting a response regression model using all the relevant covariates from the linked data. In this case, the response probability is approximated by $\hat{p}_{i}=p\left(\mathbf{x}_{i}, \hat{\beta}\right)$, where $\mathbf{x}_{i}$ is the vector of observed covariates and $\hat{\beta}$ are the regression coefficients that were estimated from fitting a response regression model to $\tilde{S}$. The resulting adjusted weights are called the propensityadjusted weights (Kim and Kim 2007). This method assumes that nonresponse is 'completely at random' given $\mathbf{x}$. We denote the propensity-adjusted weights by $w_{\mathrm{pa}}$ throughout the article.

A combination of jumpers and low response rate in the data may create additional challenges for estimating domain means. On the one hand, nonresponse adjustments are expected to further increase the $\mathrm{CV}$ of the weights in the domain of large firms, especially if the response probability is proportional to the firm's size. On the other hand, low response rates imply small effective sample sizes of the domains, which can slow down the rate of convergence of the estimators.

\section{Sample Weight Smoothing Techniques}

In this section, we adapt the two sample weight smoothing procedures, proposed by Beaumont and Rivest (2009), to the estimation of domain means in the presence of jumpers.

For this purpose, we assume that each domain (defined by the levels of $Z_{\text {surv }}$ ) is further partitioned into $T$ mutually exclusive and collectively exhaustive subdomains: $S_{\text {large }}=$ $\cup_{t=1}^{T} S_{\text {large }, t}$ and $S_{\text {small }}=\cup_{t=1}^{T} S_{\text {small }, t}$, where $S_{\text {large }}$ and $S_{\text {small }}$ are the corresponding subsets of respondents of $\tilde{S}_{\text {large }}$ and $\tilde{S}_{\text {small }}$, respectively. These subdomains can be formed by the categories of additional stratifying variables (e.g., geographic region and business sector) often used to design the sample. Analogously, $S_{\text {large }}=S_{J} \cup S_{L}$, where $S_{J}$ and $S_{L}$ are the subsets of respondents of $\tilde{S}_{J}$ and $\tilde{S}_{L}$ (defined in Section 2), respectively. Both smoothing procedures are applied independently to each subdomain $S_{\text {large }, t}=S_{J, t}$ $\cup S_{L, t}$, where $S_{J, t}$ and $S_{L, t}$ are the corresponding $t$ th subdomains of $S_{J}$ and $S_{L}(t=1, \ldots, T)$.

The first smoothing procedure defines a new weight $w_{\mathrm{BR} 1, t i}$ for each $i \in S_{\text {large }, t}$ as

$$
w_{\mathrm{BR} 1, t i}= \begin{cases}\frac{\sum_{j \in S_{\text {large }, t}} w_{t j}}{\left|S_{\text {large }, t}\right|} & \text { if } S_{J, t} \neq \emptyset \\ w_{t i} & \text { otherwise }\end{cases}
$$

where $w_{t j}$ is the final sample weight and $\left|S_{\text {large, } t}\right|$ is the size of $S_{\text {large, } t}$. It should be noted that in the presence of jumpers in $S_{\text {large, } t}$, the smoothed weight $w_{\mathrm{BR} 1, t i}$ is constant for all firms $i \in S_{\text {large, } t}$ and is equal to the average of the original weights of the jumpers and large firms in this subdomain. In other words, this procedure completely removes the variability of the weights in $S_{\text {large, } t}$ if jumpers were found in it, thus implying that all the 
firms in $S_{J, t}$ or $S_{L, t}$ represent the same number of firms in the finite population. The weights $w_{\mathrm{BR} 1}$ are referred to as BR1-weights throughout the rest of this article (BR stands for Beaumont and Rivest, who first proposed the smoothing procedure).

The BR1-weights are attractive because they are easy to implement and are suitable for multipurpose studies (like the study analyzed in Section 6) since these weights do not depend on a specific variable of interest. On the other hand, the BR1-weights of the firms in $S_{L, t}$ may become very large if the weights of the firms in $S_{J, t}$ are extremely influential (as is the case in the real data example presented in Subsection 6.3). To address this problem, Beaumont and Rivest (2009) suggested a modified smoothing procedure which consists of two steps: a trimming step and an adjustment step. In the trimming step, the weights of the jumpers in $S_{J, t}$ are trimmed using the average of the weights in $S_{\text {large, } t}$ as a trimming threshold. In other words, the weights in $S_{J, t}$ are assigned the value $w_{\mathrm{BR} 1, t}$ as defined in Equation (3). The weights in $S_{L, t}$ remain unchanged. The procedure is repeated for each $t=1, \ldots, T$ with $S_{J, t} \neq \emptyset$. In the adjustment step, a correction factor $C_{t}$ is computed as follows

$$
C_{t}= \begin{cases}\frac{\sum_{i \in S_{J, t}} w_{t i}+\sum_{i \in S_{L, t}} w_{t i}+\sum_{i \in S_{\mathrm{sma} 11, t}} w_{t i}}{\sum_{i \in S_{J, t}} w_{\mathrm{BR} 1, t i}+\sum_{i \in S_{L, t}} w_{t i}+\sum_{i \in S_{\mathrm{sma} 11, t}} w_{t i}} & \text { if } S_{J, t} \neq \emptyset \\ 1 & \text { otherwise }\end{cases}
$$

The factor $C_{t}$ is further used to adjust the weights of all the firms in $S_{\text {large, } t}$ and all the firms in the corresponding subdomain of small firms (i.e., $S_{\mathrm{small}, t}$ ) as follows

$$
w_{\mathrm{BR} 2, t i}= \begin{cases}C_{t} w_{\mathrm{BR} 1, t i} & \text { if } i \in S_{J, t} \\ C_{t} w_{t i} & \text { if } i \in S_{L, t} \text { or } i \in S_{\mathrm{Small}, t}\end{cases}
$$

We refer to these modified weights as BR2-weights. Since the original weights of the jumpers are at least as large as the BR1-weights, the coefficient $C_{t}$ is at least 1 (with equality if either $S_{J, t}=\emptyset$ or $S_{L, t}=\emptyset$ ). In practice, the value of $C_{t}$ is expected to be only slightly greater than 1 given that very few jumpers (if any) can be found in $S_{\text {large, } t}$. In this case, it follows from (5) that the modified smoothing procedure will only slightly affect the weights of the firms in $S_{L, t}$ and $S_{\mathrm{Small}, t}$, while still reducing the weights of the jumpers in $S_{J, t}$.

It is important to emphasize that the validity of the two weight smoothing procedures in Equations (3) and (5) relies on the assumption of independence between the variable of interest $y$ and the weights in a given subdomain before smoothing. In other words, the weights are required to be noninformative in that subdomain. For this reason, these smoothing procedures may not be suitable for propensity-adjusted weights, which may depend on additional covariates that can be associated with $y$, even after conditioning on $Z_{\text {surv }}$ and other characteristics that define a subdomain (e.g., geographic area and business sector). In such cases, one possible approach could be to apply the smoothing procedures to the 'cell-adjusted' weights $w_{\text {str }}$ defined in Section 2 . This option may not be feasible if only one final set of nonresponse-adjusted weights has been released to an analyst, which is often the case in practice. The independence condition will also be violated if the distribution of the $y$-variable of the jumpers is different from the 
distribution of the $y$-variable of the rest of the firms in the subdomain that the jumpers moved into. Using the BR1- and BR2-weights in situations where the conditional independence assumption is violated may lead to biased estimators. In general, parametric modeling of the weights might be necessary to smooth the weights; see Chen et al. (2017) for a detailed review.

We use $\hat{\mu}_{\mathrm{pa}}, \hat{\mu}_{\mathrm{Str}}, \hat{\mu}_{\mathrm{BR} 1}$ and $\hat{\mu}_{\mathrm{BR} 2}$ to denote the corresponding domain mean estimators obtained from Equation (2) by replacing the weights $w$ with the weights $w_{\mathrm{pa}}, w_{\mathrm{str}}, w_{\mathrm{BR} 1}$, and $w_{\mathrm{BR} 2}$, respectively. The subscript $d$ is added to emphasize the domain mean estimation where necessary.

\section{Variance Estimation}

The linearization variance estimator of the weighted mean with propensity-adjusted weights is given in the work of Kim and Kim (2007). Expanding this estimator to obtain the linearization variance estimator of the domain mean estimator $\hat{\mu}_{d}$ with nonresponseadjusted weights, defined in the previous sections, can be challenging due to the presence of jumpers and weight smoothing. Replication methods, in particular bootstrap variance estimators, are recommended in these cases (Mashreghi et al. 2016). Beaumont and Rivest (2009) used the rescaled bootstrap method (Rao and Wu 1988; Rao et al. 1992) to estimate the variance of the population total estimator obtained from a stratified sample of firms. The formula for bootstrap (rescaled) weights that account for sampling without replacement from a finite population is given in Beaumont and Patak 2012. In this study, we used the 'doubled half' bootstrap method devised by Antal and Tillé (2014) for sampling designs without replacement.

\subsection{Bootstrap Variance Estimator}

Let $\tilde{S}$ be a stratified random sample from the finite population of firms. Assume that the information about the response status of each firm is contained in $\tilde{S}$ as a binary variable response. This variable takes value 1 for respondents and 0 otherwise. The following steps describe the implementation of the method for estimating the variances of $\hat{\mu}_{d}$, calculated using the nonresponse-adjusted weights defined in the previous sections, with or without smoothing.

1. Obtain a bootstrapped sample $\tilde{S}^{*}$ from $\tilde{S}$ by applying the 'doubled half' method to each stratum of $\tilde{S}$.

2. The sample $\tilde{S}^{*}$ inherits the response status contained in the variable response from the parent sample $\tilde{S}$.

3. Estimate the response probabilities $\hat{p}_{i}^{*}$ from $\tilde{S}^{*}$ (for example, using a logistic regression model).

4. A subset of $\tilde{S}^{*}$, for which the value of response is equal to 1 , is used as a bootstrap replicate $S^{*}$ of a sample of respondents $S$. Calculate $w_{\mathrm{pa}, i}^{*}, w_{\mathrm{Str}, i}^{*}, w_{\mathrm{BR} 1, i}^{*}$ and $w_{\mathrm{BR} 2, i}^{*}$ for each $i \in S^{*}$.

5. Estimate the bootstrap domain means $\mu_{d}^{*}$ using one of the estimators: $\hat{\mu}_{\text {pa }}^{*}, \hat{\mu}_{\text {str }}^{*}$, $\hat{\mu}_{\mathrm{BR} 1}^{*}$ and $\hat{\mu}_{\mathrm{BR} 2}^{*}$. 
6. Repeat steps $1-5 R$ times to obtain a sequence of bootstrap estimates

$$
\hat{\mu}_{d}^{*(1)}, \hat{\mu}_{d}^{*(2)}, \ldots, \hat{\mu}_{d}^{*(R)}
$$

for each of the four estimators.

7. Estimate $V\left(\hat{\mu}_{d}\right)$ as

$$
\hat{V}\left(\hat{\mu}_{d}\right)=\frac{1}{R-1} \sum_{r=1}^{R}\left(\hat{\mu}_{d}^{*(r)}-\overline{\hat{\mu}}_{d}\right)^{2}
$$

where $\overline{\hat{\mu}}_{d}=\frac{1}{R} \sum_{r=1}^{R} \hat{\mu}_{d}^{*(r)}$.

\section{Application to an Organizational Performance Survey}

In this section we illustrate domain mean estimation using data from the Ontario Leading Indicators Project (OLIP) survey. OLIP is a cross-sectional business survey designed for auditing the organizational performance in preventing work-related injury and illness using reliable and validated indicators (measures) (Institute for Work and Health 2011). The target population consists of firms in Ontario, Canada, that were registered with the Workplace Safety and Insurance Board (WSIB, http://www.wsib.on.ca/), an organization responsible for workers' compensation. The number of full-time equivalents (FTEs), derived from the WSIB payroll information, was used as the measure of firm size at the design stage. Only firms with at least one FTE in 2009, and in one of the following industries were inluded: agriculture, manufacturing, service, education, municipal, healthcare, pulp and paper, construction, transportation, electrical and utilities (Institute for Work and Health 2011).

Respondents were asked to classify the size of their firm, $Z_{\text {surv }}$, into five categories:

$$
Z_{\text {surv }, i}= \begin{cases}\text { very small } & \text { if firm } i \text { has } 1 \text { to } 4 \text { employees } \\ \text { small } & \text { if firm } i \text { has } 5 \text { to } 19 \text { employees } \\ \text { medium } & \text { if firm } i \text { has } 20 \text { to } 99 \text { employees } \\ \text { large } & \text { if firm } i \text { has } 100 \text { to } 299 \text { employees } \\ \text { very large } & \text { if firm } i \text { has } 300 \text { or more employees }\end{cases}
$$

Considering each level of $Z_{\mathrm{surv}}$ as a domain in the target population, the focus here is on estimating the population domain means and their $95 \%$ confidence intervals (CI) for six variables of interest: Safety Practices (SP), Health and Safety Leadership (HSL), Ergonomic Practices (EP), Disability Prevention (DP), People-Oriented Culture (POC), and Organizational Performance Measure (OPM). These variables were derived from the key OHS measures collected by the OLIP study (Institute for Work and Health 2013). Each measure was presented in the questionnaire as a scale with a variable number of items. Each item had five possible responses, with values from 0 to 4 , with higher values corresponding to better performance. The individual value of a final variable of interest was obtained by taking the average of individual responses on the items within a given scale, resulting in a continuous variable in the range $[0 ; 4]$. 
The selected sample $(\tilde{S})$ was designed as a stratified simple random sample, with the strata formed by two levels of the firm size $Z_{\text {des }}$ derived from the FTE data in 2009 (sma 11 if the firm has 1 to 19 employees and large if the firm has 20 or more employees), five geographical regions in Ontario, and ten business sectors as reported on the WSIB data registry, resulting in a total of 100 strata. For agriculture, manufacturing and service sectors, 300 firms were randomly sampled from a stratum. In the other seven sectors, 150 firms were sampled randomly from the stratum. Otherwise, all firms in the stratum were sampled. Base weights $(b)$ were calculated from these sampling fractions. The total size of $\tilde{S}$ was $\tilde{n}=12,767$, of which $n=1,639$ were respondents, indicating a low response rate $(\approx$ $13 \%$ ). Finally, $\tilde{S}$ was linked to the WSIB administrative data at the firm level, which allowed us to obtain information about compensation claims activity for all firms in $\tilde{S}$.

\subsection{Nonresponse Adjustment}

The nonresponse-adjusted weights $w_{\mathrm{str}}$ and $w_{\mathrm{pa}}$ have been calculated as described in Section 3. Results from the logistic regression model, fitted with and without base weights $(b)$ to obtain $w_{\text {pa }}$, were nearly identical (Kim and Kim 2007). Three design variables $\left(Z_{\text {des }}\right.$, geographic region and business sector) along with various covariates related to firms' claims activity were included in the response regression model. The estimated regression coefficient of $Z_{\text {des }}$ was 0.76 , which indicates higher response probabilities for large firms, after adjusting for all the other factors. Firms in municipal, electrical and utilities, and construction sectors had the highest negative coefficients $(-1.15,-1.15$ and -1.14 , respectively), which indicates the lowest response probabilities for these sectors. Interestingly, firms with higher claim rates showed higher response probabilities: the regression coefficient was equal to 0.14 . All five coefficients had $p$-values smaller than 0.001 .

We validated the nonresponse-adjusted weights by comparing the weighted estimators from $\tilde{S}$ using the weights $b$ (complete data case) with the weighted estimators from $S$ using the weights $b$ (and thus completely ignoring the nonresponse), $w_{\mathrm{str}}$, and $w_{\mathrm{pa}}$, respectively, for a number of variables in $\tilde{S}$ (Lohr et al. 2016). Both nonresponse-adjusted estimators showed closer correspondence with the complete case estimates when compared to the unadjusted estimator (the details are available from the corresponding author).

\subsection{Application of Smoothing Procedures for $\mathrm{D}=5$}

In OLIP, the five levels of $Z_{\text {surv }}$ define $D=5$ domains of interest: very sma 11 , small, medium, large, and very large, which can also be denoted using the notation in Section 4 as $S_{\text {very small }}, S_{\text {small }}, S_{\text {medium }}, S_{\text {large, }}$ and $S_{\text {very large, }}$ respectively. Five geographic regions and ten business sectors partition each of the five domains into 50 subdomains. The firms that were registered as small firms in the WSIB data $\left(Z_{\text {des }}=\right.$ smal1) but reported more than 19 employees in the survey were declared jumpers. Using this definition, 45 jumpers were found in $S_{\text {medium }}, 12$ in $S_{\text {large }}$, and 9 in $S_{\text {very large }}$ - for a total of 66 jumpers.

Weight smoothing procedures described in Section 4 were adapted to each subdomain of medium, large, and very large firms in which jumpers were found. For example, for a given $t$, if jumpers were found in $S_{\text {medium, } t}$ and $S_{\text {large, } t}$, but not in $S_{\text {very large, } t}$, the BR1-weights were computed by applying Equation (3) independently to $S_{\text {medium, } t}$ and 
$S_{\text {large, } t}$. The corresponding BR1-weight was then used as a trimmimg threshold to complete the trimming step of the BR2-procedure as described in Section 4. Next, letting $S_{\text {medium }, t}=S_{J_{1}, t} \cup S_{M, t}$ and $S_{\text {large }, t}=S_{J_{2}, t} \cup S_{L, t}$, the correction factor was obtained analogously applying to Equation (4) as

$$
\frac{\sum_{i \in S_{J_{1}, t}} w_{t i}+\sum_{i \in S_{J_{2}, t}} w_{t i} \sum_{i \in S_{M, t}} w_{t i}+\sum_{i \in S_{L, t}} w_{t i}+\sum_{i \in S_{\text {very }}} w_{t i}+\sum_{i \in S_{\text {sma } 11, t}, t} w_{t i}}{\sum_{i \in S_{J_{1}, t}} w_{\mathrm{BR} 1, t i}+\sum_{i \in S_{J_{2}, t}} w_{\mathrm{BR} 1, t i} \sum_{i \in S_{M, t}} w_{t i}+\sum_{i \in S_{L, t}} w_{t i}+\sum_{i \in S_{\text {very }} \text { smal1 }, t} w_{t i}+\sum_{i \in S_{\mathrm{Smal1}, t}} w_{t i}}
$$

Finally, the weights of the firms in $S_{\text {very small, } t}, S_{\mathrm{small}, t}, S_{\text {medium }, t}$ and $S_{\text {large, } t}$ were updated as in Equation (5). The weights of the firms in $S_{\text {very large, } t}$ remained unchanged since no jumpers were found in this subdomain.

In this example we defined jumpers as firms that were registered as $\operatorname{small}\left(Z_{\text {des }}=\right.$ smal1) but reported their size in the survey as medium, large or very large $\left(Z_{\text {surv }}=\right.$ medium, large, very large $)$. It is worth noting that this definition of jumpers is not fixed and can be adjusted depending on the particular problem. For example, we performed a sensitivity analysis using another definition, by which the firms defined as $\left(Z_{\text {des }}=\operatorname{smal1}, Z_{\text {surv }}=\right.$ medium $)$ were not declared jumpers (45 firms in this example). The resulting means in the domain of medium firms were virtually identical under both definitions (data not shown), since the weights of these 45 firms were not extreme for the vast majority of cases. The flexibility in defining jumpers may also be useful if the categories of the variable $Z_{\text {surv }}$ do not allow a clear-cut partition into small and large firms as defined by the categories of the variable $Z_{\text {des }}$. In general, we recommend exploring the distribution of the weights in the domains defined by $Z_{\text {surv }}$ before making a decision about the partition of the firms into small and large to confirm that the proposed definition of jumpers is sensible for a given problem. Once jumpers have been identified, the smoothing procedures described in the previous sections should be applied to the domains (defined by $Z_{\text {surv }}$ ) that contain jumpers.

\subsection{Estimation of Domain Means by Firm Size}

In this section we illustrate the estimation of the domain means of large and very large firms. The number of respondents in each of these domains were 260 and 166, respectively.

The CV of the base and nonresponse-adjusted weights, with and without smoothing, are shown in Table 1 . The smoothed weights, $w_{\mathrm{BR} 1}$ and $w_{\mathrm{BR} 2}$, were applied to the cell-adjusted

Table 1. The coefficient of variation $(C V, \%)$ of base $(b)$, propensity-adjusted $\left(w_{p a}\right)$, celladjusted $\left(w_{s t r}\right)$, and two sets of smoothed weights $\left(w_{B R 1}, w_{B R 2}\right)$ in the domain of large and very large firms in the OLIP sample. The smoothed weights were calculated by applying the smoothing procedures defined in Equations (3) and (5) to the cell-adjusted weights $\left(w_{\text {str }}\right)$.

\begin{tabular}{lccccc}
\hline & \multicolumn{5}{c}{ Weights } \\
\cline { 2 - 6 } Domain & $b$ & $w_{\mathrm{pa}}$ & $w_{\mathrm{str}}$ & $w_{\mathrm{BR} 1}$ & $w_{\mathrm{BR} 2}$ \\
\hline Large & 156.3 & 341.5 & 513.2 & 246.6 & 586.6 \\
Very large & 265.6 & 642.3 & 581.6 & 294.1 & 353.4 \\
\hline
\end{tabular}


weights $w_{\text {str }}$ to ensure that the conditional independence assumption is met. The numbers in the table indicate the impact of the jumpers on the $\mathrm{CV}$ of the base weights, an additional increase in the $\mathrm{CV}$ caused by the nonresponse adjustment, and the impact of the two smoothing procedures on reduction in the $\mathrm{CV}$.

Figures 1 and 2 (Online supplemental data) show the estimated means and their 95\% CIs for the domain of large and very large firms, respectively. To ensure that the lower and upper bounds of the CIs lie within the range [0;4], we applied a logit-type transformation $g(\hat{\mu})=\frac{\hat{\mu}}{4-\hat{\mu}}$, similarly to a commonly used approach for proportions (Lachin 2011). The resulting CIs for $\hat{\mu}$ are almost identical to the symmetric CIs obtained without a transformation, except for a few cases where the estimated upper bounds of the symmetric CIs were above 4 . In these few cases, the resulting CIs are noticeably asymmetric.

Overall, both nonresponse-adjusted estimators (without smoothing) produced very similar estimates for the majority of study variables in both domains. This may reflect the fact that the design variables were major predictors for response. The discrepancies between the estimates with and without smoothing of the weights can be seen in some $y$-variables, most noticeably in the OPM variable in the domain of very large firms. A substantially lower domain mean estimate $\hat{\mu}_{\mathrm{BR} 1}$ was obtained for EP in the domain of very large firms (see Online supplemental data, Figure 2). With further checking we found that this dramatic difference in the estimated mean was caused by a single observation that corresponded to a very large firm with a very small value of EP and a very large BR1-weight. It can also be seen from the graph that the estimator with BR2weights nicely addressed this problem. In the next section a simulation study is used to compare the performance of the weighted estimators in terms of bias and efficiency.

\section{Simulation Study}

The extensive simulation study described in this section was designed with two goals in mind. First, we hypothesized that two parameters of the finite population, namely, (1) $p_{\text {jump }}$ the percent of jumpers among small firms, and (2) $\delta$ - the difference between the mean of the $y$-values of the jumpers and the mean of the $y$-values of the firms in the subdomain they jumped into, may have a considerable impact on the performance of the weighted estimators. In particular, we anticipated that the smoothed estimators will be biased when $\delta>0$, since in this case the assumption of independence between the weights and the $y$-variable within a subdomain fails. We also expected that the increase in $p_{\text {jump }}$ may improve the performance of the estimators. Therefore, we created different scenarios to explore the performance of the estimators for various combinations of $\delta$ and $p_{\text {jump. }}$. Second, we aimed to test the performance of Antal-Tille's bootstrap variance estimator, described in Section 5 and used in the analysis of the OLIP data. We also studied the error rate of the CIs constructed from the bootstrap estimates.

\subsection{Data Generation}

\subsubsection{Generation of a Finite Population}

The finite population of firms was reconstructed from the selected sample $\tilde{S}$ in the OLIP study by repeating each row in $\tilde{S}$ as many times as its base weight $b$. A 
continuous variable for FTEs in 2009 was used to construct the variables $Z_{\text {des }}$ and $Z_{\text {surv }}$ as defined in Section 6. Five geographic regions in Ontario, $\left\{G_{1}, \ldots, G_{5}\right\}$, were used together with the two categories of $Z_{\text {des }}$ to form 10 design strata, which can be enumerated in pairs: for example, the pair $\left(\operatorname{small}, G_{1}\right)$ corresponds to the stratum of small firms in region $\mathrm{G}_{1}$. Let $N$ and $N_{h}(h=1, \ldots, 10)$ be the sizes of the finite population and the stratum $h$, respectively, as defined by design. The values of the $y$ variable were generated independently within each stratum $h$ from a normal distribution with mean $\mu_{h}$ and a common standard deviation of 0.1 . The values of $\mu_{h}$ were chosen such that the large firms have higher mean values than the small firms. For illustration, we estimate the mean of the $y$-variable in the domain of very large firms $\left(Z_{\text {surv }}=\right.$ very large $)$.

\subsubsection{Generation of Jumpers in the Population}

First, $p_{\text {jump }}(\%)$ of the firms from all the small firms in the population $\left(Z_{\text {des }}=\right.$ smal1) were randomly assigned to be jumpers. For simplicity, we assumed that all the generated jumpers changed their firm size from small to very large and changed the original value of $Z_{\text {surv }}$ for these firms to very large. Next, the values of the $y$-variable of the jumpers were re-generated from a normal distribution with mean $\mu_{h} *+\delta$ and standard deviation 0.1 , where $\mu_{h}{ }^{*}$ corresponds to the mean used to create the $y$-values of large firms from the same geographic region and $\delta \geq 0$ is a predefined constant. We set $\delta \in\{0,0.15,0.25,0.5\}$ to reflect various scenarios when the mean of the $y$-variable of the jumpers is up to $12.5 \%$ greater than the mean of the $y$-variable of the large firms. The assumption of conditional independence between the $y$-variable and the weights in a given subdomain holds when $\delta=0$. Scenarios with $\delta>0$ reflect the fact that jumpers may be very different from the firms in the subdomain they jumped into. The assumption of conditional independence is not met in this case and the results will serve as sensitivity analyses. We set $p_{\text {jump }} \in\{1 \%$, $2 \%, 5 \%, 7 \%, 10 \%$ \} to reflect that in most practical cases the percent of jumpers in the population is not expected to be greater than $10 \%$ (Favre-Martinoz et al. 2015). Since approximately $88 \%$ of the firms in the artificially created population have $Z_{\text {des }}=$ smal1, these values reflect most practical scenarios fairly well. All possible combinations of $p_{\text {jump }}$ and $\delta$ define 20 scenarios of interest.

\subsubsection{Obtaining a Sample of Respondents}

Business surveys are prone to nonresponse with variable response rates. US government business surveys that require mandatory participation, can have a response rate as high as $80 \%$ or higher (U.S. Department of Labor 2019). Low response rates (under 50\%) might be expected in smaller surveys, in which participation is not mandatory (Cook et al. 2009). In this study, we decided to explore the properties of the three weighted estimators under a low response rate $(20 \%)$ and a target response rate $(80 \%)$. A sample of respondents was selected from the finite population in a two-step process. First, a full sample $\tilde{S}$ was selected as a stratified random sample without replacement with sample inclusion probabilities equal to $\tilde{n}_{h} / N_{h}$ for predetermined $\tilde{n}_{h}$ (see Table 2). The values of $\tilde{n}_{h}$ were selected to allow sufficient variation in sample weights between the strata of small and large firms. In the 
Table 2. Numerical values of the parameters used in the data generation process: $N_{h}-$ the hth stratum size in the population; $\tilde{n}_{h}$ - the hth stratum size in the selected sample $\tilde{S} ; b_{h}=N_{h} / \tilde{n}_{h}$ (rounded to a whole number); $\mu_{h}$ - the mean of the $y$-variable in the hth stratum in the population.

\begin{tabular}{lcrrr}
\hline Design stratum & $N_{h}$ & $\tilde{n}_{h}$ & $b_{h}$ & $\mu_{h}$ \\
\hline$\left(\right.$ small,$\left.G_{1}\right)$ & 62,938 & 252 & 250 & 2.3 \\
$\left(\right.$ small,$\left.G_{2}\right)$ & 36,826 & 246 & 150 & 2.0 \\
$\left(\right.$ small,$\left.G_{3}\right)$ & 29,164 & 243 & 120 & 1.8 \\
$\left(\right.$ small,$\left.G_{4}\right)$ & 24,273 & 405 & 60 & 2.1 \\
$\left(\right.$ small,$\left.G_{5}\right)$ & 13,960 & 465 & 30 & 2.5 \\
$\left(\right.$ large,$\left.G_{1}\right)$ & 9,468 & 947 & 10 & 3.6 \\
$\left(\right.$ large,$\left.G_{2}\right)$ & 4,651 & 1,163 & 4 & 3.4 \\
$\left(\right.$ large,$\left.G_{3}\right)$ & 4,864 & 973 & 5 & 3.2 \\
$\left(\right.$ large, $\left.G_{4}\right)$ & 2,734 & 1,367 & 2 & 3.5 \\
$\left(\right.$ large,$\left.G_{5}\right)$ & 1,478 & 1,478 & 1 & 3.8 \\
\hline
\end{tabular}

Note: (small, $G_{1}$ ) refers to a stratum of small firms in the geographic region $G_{1}$.

second step, the sample of respondents was selected using Poisson sampling with two sets of response probabilities $p_{i}$ that correspond to the two response rates:

$$
\operatorname{logit}\left(p_{i}\right)= \begin{cases}-3.5+0.5 z_{i}+\mathrm{g}_{1 i}+\mathrm{g}_{2 i}+\mathrm{g}_{4 i}+\mathrm{g}_{5 i} & \text { for response rate }=20 \% \\ -0.45+0.5 z_{i}+\mathrm{g}_{1 i}+\mathrm{g}_{2 i}+\mathrm{g}_{4 i}+\mathrm{g}_{5 i} & \text { for response rate }=80 \%\end{cases}
$$

where $z$ is the firm size variable; the variables $g_{1}, g_{2}, g_{4}, g_{5}$ are dummy variables for the geographic regions $G_{1}, G_{2}, G_{4}, G_{5}$, respectively $\left(G_{3}\right.$ was defined as a reference); and $\operatorname{logit}\left(p_{i}\right)=\frac{p_{i}}{1-p_{i}}$.

\subsection{Performance of the Weighted Estimators}

We examined the performance of the weighted estimators with propensity-adjusted weights, with and without smoothing, for estimating the mean of the $y$-variable in the domain of very large firms $\left(Z_{\text {surv }}=\right.$ very large $)$ under 20 scenarios of interest and two response rates: $20 \%$ and $80 \%$. The estimated response probabilities $\hat{p}_{i}$, used to construct the propensityadjusted weights $w_{\text {pa }}$, were obtained by fitting the logistic regression model with firm size and region as regressors. The smoothing procedures were applied to the propensity-adjusted weights.

The simulations were repeated 5,000 times for each scenario. In scenarios with $p_{\text {jump }}=0 \%$, the average sample size of the domain of very large firms is 140 and 290 under a $20 \%$ and an $80 \%$ response rate, respectively. In scenarios with $p_{\text {jump }}=1 \%$, the average number of jumpers equals 2 and 12 under a $20 \%$ and an $80 \%$ response rate, respectively. In scenarios with $p_{\text {jump }}=10 \%$, the average number of jumpers equals 17 and 115 under a $20 \%$ and an $80 \%$ response rate, respectively.

The $\mathrm{CV}$ of the weights, relative bias (RB) and relative efficiency (RE) were computed for each scenario. The reported $\mathrm{CV}$ of the weights is the average of the 5,000 ratios of the standard deviation of the weights to their mean obtained from each simulated set. The RE of the estimators with smoothed weights was computed relative to the estimator with propensity-adjusted weights. 


\subsection{1. $\mathrm{CV}$ of the Weights}

It follows from the results in Table 3, that the CV of $w_{\mathrm{pa}}$ is decreasing with the increase in $p_{\text {jump. }}$. In contrast, the $\mathrm{CV}$ of $w_{\mathrm{BR} 2}$ is increasing with the increase in $p_{\text {jump. }}$. The $\mathrm{CV}$ of $w_{\mathrm{BR} 1}$ stays almost constant for different values of $p_{\text {jump. }}$ Both BR1- and BR2-weights have lower $\mathrm{CV}$ than the propensity-adjusted weights. The $\mathrm{CV}$ of the weights is similar for both response rates as expected.

\subsection{2. $\mathrm{RB}$ and $\mathrm{RE}$ of the Estimators when $\delta=0$}

The RB and RE of the estimators when $\delta=0$ are summarized in Tables 4-5. The results in Table 4 show that the RB of $\hat{\mu}_{\mathrm{pa}}$ and $\hat{\mu}_{\mathrm{BR} 1}$ is close to zero for all values of $p_{\text {jump }}$ under a $20 \%$ response rate. The $\mathrm{RB}$ of the two estimators is essentially equal to zero under an $80 \%$ response rate. The $\mathrm{RB}$ of $\hat{\mu}_{\mathrm{BR} 2}$ changes from negative to positive with the increase in $p_{\text {jump. }}$ This pattern is similar for both response rates with a somewhat noticeable shift in the value of $p_{\text {jump }}$ at which the RB crosses zero. Though the values of the RB of $\hat{\mu}_{\mathrm{BR} 2}$ are higher than the values of the RB of the other two estimators, the increase in bias is not alarming. As follows from the results in Table 5, $\hat{\mu}_{\mathrm{BR} 1}$ is more efficient than $\hat{\mu}_{\mathrm{pa}}$ for any value of $p_{\text {jump }}$ and for both response rates. Similarly, $\hat{\mu}_{\mathrm{BR} 2}$ is more efficient than $\hat{\mu}_{\mathrm{pa}}$ for any value of $p_{\text {jump }}$ that we studied under a $20 \%$ response rate. However, under a higher response rate, $\hat{\mu}_{\mathrm{BR} 2}$ is less efficient than $\hat{\mu}_{\mathrm{pa}}$ for $p_{\text {jump }}>5 \%$.

\subsection{3. $\mathrm{RB}$ and RE of the Estimators when $\delta>0$}

Tables 4 and 5 also contain the RB and RE of the estimators when $\delta=0.15$. The full results of the sensitivity analyses that compare the RB and RE of the estimators for $\delta \in\{0$, $0.15,0.25,0.5\}$ are presented in Online Supplemental data, Figures 3-7: Figure 3 displays the RBs of $\hat{\mu}_{\mathrm{pa}}$, Figures 4-5 display the RBs of $\hat{\mu}_{\mathrm{BR} 1}$ and $\hat{\mu}_{\mathrm{BR} 2}$, and Figures $6-7$ display the REs of $\hat{\mu}_{\mathrm{BR} 1}$ and $\hat{\mu}_{\mathrm{BR} 2}$, respectively. For lower values of $p_{\text {jump }}(<5 \%)$, the RB of $\hat{\mu}_{\mathrm{pa}}$ increases with the increase in $\delta$ under a $20 \%$ response rate. However, as $p_{\text {jump }}$ increases, the bias stabilizes around 0 . Under a higher response rate, the bias of $\hat{\mu}_{\mathrm{pa}}$ is relatively small for all values of $\delta$. The bias of $\hat{\mu}_{\mathrm{BR} 1}$ stays almost constant for different values of $p_{\text {jump }}$ and increases with the increase in $\delta$. The bias of $\hat{\mu}_{\mathrm{BR} 2}$ also increases with the

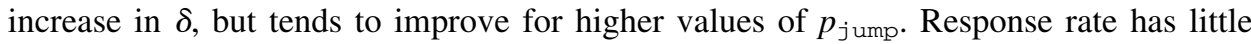
effect on the bias of the smoothed estimators. The RE of the smoothed estimators tends to increase as $\delta$ increases. The bias constitutes a major part of this increase. The informativeness of the weights in the subdomains, in which the smoothing was applied, is the reason for this bias: the distribution of the $y$-variable depends on the values of the design variable $Z_{\text {des }}$ when $\delta>0$, thus violating the conditional independence assumption.

\subsection{Performance of Antal-Tille's Bootstrap Variance Estimator}

To evaluate the performance of Antal-Tille's bootstrap estimator, we implemented the method with $R=500$ bootstraps for each of the 500 samples simulated as described in Subsection 7.1. The reduction in the number of simulations was needed to reduce the time of computation. To check that the 500 simulated sets are sufficient, we compared the values of the sampling standard errors (SSEs) (defined as the standard deviation of the estimated domain means) of the estimators from 500 versus 5,000 simulated sets and 
Table 3. Average coefficient of variation $(C V, \%)$ of $w_{p a}, w_{B R 1}$ and $w_{B R 2}$ under a $20 \%$ and an $80 \%$ response rate.

\begin{tabular}{|c|c|c|c|c|c|c|}
\hline \multirow[b]{2}{*}{ Response rate } & \multirow[b]{2}{*}{ Weight } & \multicolumn{5}{|c|}{$p_{\text {jump }}, \%$} \\
\hline & & 1 & 2 & 5 & 7 & 10 \\
\hline \multirow{3}{*}{$20 \%$} & $w_{\mathrm{pa}}$ & 400.5 & 471.0 & 433.1 & 392.3 & 345.2 \\
\hline & $w_{\mathrm{BR} 1}$ & 85.3 & 87.1 & 83.0 & 79.4 & 74.5 \\
\hline & $w_{\mathrm{BR} 2}$ & 90.1 & 108.4 & 161.1 & 185.5 & 205.4 \\
\hline \multirow{3}{*}{$80 \%$} & $w_{\mathrm{pa}}$ & 329.8 & 314.1 & 247.1 & 221.0 & 194.2 \\
\hline & $w_{\mathrm{BR} 1}$ & 64.7 & 66.9 & 64.2 & 64.2 & 63.6 \\
\hline & $w_{\mathrm{BR} 2}$ & 73.6 & 93.6 & 138.4 & 150.3 & 151.8 \\
\hline
\end{tabular}

Note: $p_{\text {jump }}$ - percent of jumpers among small firms in the finite population.

Table 4. Relative bias $(R B, \%)$ of $\hat{\mu}_{p a}, \hat{\mu}_{B R 1}$ and $\hat{\mu}_{B R 2}$ under a $20 \%$ and an $80 \%$ response rate.

\begin{tabular}{|c|c|c|c|c|c|c|c|}
\hline \multirow[b]{2}{*}{ Response rate } & \multirow[b]{2}{*}{$\delta$} & \multirow[b]{2}{*}{ Estimator } & \multicolumn{5}{|c|}{$p_{\text {jump }}, \%$} \\
\hline & & & 1 & 2 & 5 & 7 & 10 \\
\hline $20 \%$ & 0.15 & $\begin{array}{l}\hat{\mu}_{\mathrm{pa}} \\
\hat{\mu}_{\mathrm{BR} 1} \\
\hat{\mu}_{\mathrm{BR} 2} \\
\hat{\mu}_{\mathrm{pa}} \\
\hat{\mu}_{\mathrm{BR} 1} \\
\hat{\mu}_{\mathrm{BR} 2}\end{array}$ & $\begin{array}{l}-0.052 \\
-0.048 \\
-0.737 \\
-0.567 \\
-2.231 \\
-2.844\end{array}$ & $\begin{array}{r}0.108 \\
0.138 \\
-0.839 \\
-0.216 \\
-2.718 \\
-3.266\end{array}$ & $\begin{array}{r}0.127 \\
0.143 \\
-0.499 \\
-0.001 \\
-3.125 \\
-2.578\end{array}$ & $\begin{array}{r}0.136 \\
0.146 \\
-0.184 \\
-0.055 \\
-3.196 \\
-1.933\end{array}$ & $\begin{array}{r}0.097 \\
0.061 \\
0.168 \\
-0.056 \\
-3.251 \\
-1.144\end{array}$ \\
\hline $80 \%$ & 0.15 & $\begin{array}{l}\hat{\mu}_{\mathrm{pa}} \\
\hat{\mu}_{\mathrm{BR} 1} \\
\hat{\mu}_{\mathrm{BR} 2} \\
\hat{\mu}_{\mathrm{pa}} \\
\hat{\mu}_{\mathrm{BR} 1} \\
\hat{\mu}_{\mathrm{BR} 2}\end{array}$ & $\begin{array}{r}0.007 \\
-0.024 \\
-0.665 \\
-0.127 \\
-2.135 \\
-2.556\end{array}$ & $\begin{array}{l}-0.008 \\
-0.009 \\
-0.592 \\
-0.060 \\
-2.672 \\
-2.594\end{array}$ & $\begin{array}{r}-0.019 \\
-0.020 \\
0.150 \\
-0.058 \\
-2.955 \\
-1.313\end{array}$ & $\begin{array}{r}0.003 \\
-0.050 \\
0.441 \\
0.016 \\
-2.874 \\
-0.594\end{array}$ & $\begin{array}{r}0.008 \\
-0.008 \\
0.700 \\
-0.002 \\
-2.719 \\
0.073\end{array}$ \\
\hline
\end{tabular}

Note: $\delta$ - difference between the mean of the $y$-values of the jumpers and the mean of the $y$-values of the rest of the firms in the subdomain, in the finite population; $p_{\text {jump }}$ - percent of jumpers among small firms in the finite population.

Table 5. Relative efficiency $(R E, \%)$ of $\hat{\mu}_{B R 1}$ and $\hat{\mu}_{B R 2}$ under a $20 \%$ and an $80 \%$ response rate.

\begin{tabular}{|c|c|c|c|c|c|c|c|}
\hline \multirow[b]{2}{*}{ Response rate } & \multirow[b]{2}{*}{$\delta$} & \multirow[b]{2}{*}{ Estimator } & \multicolumn{5}{|c|}{$p_{\text {jump }}, \%$} \\
\hline & & & 1 & 2 & 5 & 7 & 10 \\
\hline \multirow{4}{*}{$20 \%$} & 0 & $\hat{\mu}_{\mathrm{BR} 1}$ & 69 & 74 & 75 & 75 & 75 \\
\hline & & $\hat{\mu}_{\mathrm{BR} 2}$ & 21 & 26 & 37 & 48 & 72 \\
\hline & 0.15 & $\hat{\mu}_{\mathrm{BR} 1}$ & 126 & 184 & 317 & 386 & 492 \\
\hline & & $\hat{\mu}_{\mathrm{BR} 2}$ & 131 & 188 & 208 & 180 & 140 \\
\hline \multirow{4}{*}{$80 \%$} & 0 & $\hat{\mu}_{\mathrm{BR} 1}$ & 72 & 70 & 72 & 76 & 76 \\
\hline & & $\hat{\mu}_{\mathrm{BR} 2}$ & 53 & 59 & 74 & 140 & 266 \\
\hline & 0.15 & $\hat{\mu}_{\mathrm{BR} 1}$ & 401 & 745 & 1,717 & 2,143 & 2,653 \\
\hline & & $\hat{\mu_{\mathrm{BR} 2}}$ & 503 & 675 & 429 & 211 & 134 \\
\hline
\end{tabular}

Note: $\delta$ - difference between the mean of the $y$-values of the jumpers and the mean of the $y$-values of the rest of the firms in the subdomain, in the finite population; $p_{\text {jump }}$ - percent of jumpers among small firms in the finite population. 
obtained virtually identical values in both cases. Next, we computed the estimated standard errors (ESEs) (defined as the average of the 500 estimated standard errors) and the ratios SSE/ESE. Ratios close to 1 indicate good performance of the bootstrap variance estimator, whereas ratios greater than 1 indicate variance underestimation. In addition, the 95\% CIs around the estimated domain means were constructed using the $2.5 \%$ and $97.5 \%$ percentiles of the bootstrap distribution obtained for each simulated dataset. The error rate (ER), defined as the number of times in which the true value of the domain mean was not included in the corresponding CI, was computed.

In the absence of jumpers (i.e., $p_{\text {jump }}=0 \%$ ), the SSE/ESE ratios of $\hat{\mu}_{\mathrm{pa}}$ are 1.0123 and 1.0228 under a $20 \%$ and an $80 \%$ response rates, respectively, which are close to 1 , as expected. The SSE/ESE ratios for $p_{\text {jump }} \in\{1 \%, 2 \%, 5 \%, 7 \%, 10 \%\}$ are presented in Table 6. As we can see from these results, the values of the SSE/ESE ratio for $\hat{\mu}_{\mathrm{BR} 2}$ are close to 1 under both response rates. Also, the SSE/ESE ratios are very close to 1 for all three estimators under an $80 \%$ response rate, thus confirming a good performance of the bootstrap variance estimator in these cases. Under a $20 \%$ response rate, the variances of $\hat{\mu}_{\mathrm{pa}}$ and $\hat{\mu}_{\mathrm{BR} 1}$ are underestimated for low values of $p_{\text {jump }}$, regardless of the value of $\delta$.

In the absence of jumpers (i.e., $p_{\text {jump }}=0 \%$ ), the ER values of $\hat{\mu}_{\mathrm{pa}}$ are $5.4 \%$ and $5.8 \%$ under a $20 \%$ and an $80 \%$ response rates, respectively, which are close to the nominal $5 \%$, as expected. The ERs for $p_{\text {jump }} \in\{1 \%, 2 \%, 5 \%, 7 \%, 10 \%\}$ are presented in Table 7 . The ERs of $\hat{\mu}_{\mathrm{pa}}$ improve considerably and get closer to the nominal $5 \%$ with the increase in $p_{\text {jump }}$ and under a higher response rate. The ERs of the smoothed estimators seem to be seriously affected by the value of $\delta$ under both response rates. The increase in bias and distortion of the sampling distribution (see Online supplemental data, Figures 8-11) can potentially explain these abnormally high ERs.

Table 6. The SSE/ESE ratio of $\hat{\mu}_{p a}, \hat{\mu}_{B R 1}$ and $\hat{\mu}_{B R 2}$ under a $20 \%$ and an $80 \%$ response rate.

\begin{tabular}{|c|c|c|c|c|c|c|c|}
\hline \multirow[b]{2}{*}{ Response rate } & \multirow[b]{2}{*}{$\delta$} & \multirow[b]{2}{*}{ Estimator } & \multicolumn{5}{|c|}{$p_{\text {jump }}, \%$} \\
\hline & & & 1 & 2 & 5 & 7 & 10 \\
\hline \multirow{7}{*}{$20 \%$} & \multirow{3}{*}{0} & $\hat{\mu}_{\mathrm{pa}}$ & 1.4297 & 1.3439 & 1.1343 & 1.1031 & 1.0310 \\
\hline & & $\hat{\mu}_{\mathrm{BR} 1}$ & 1.3851 & 1.2969 & 1.1424 & 1.0841 & 1.0205 \\
\hline & & $\hat{\mu}_{\mathrm{BR} 2}$ & 1.0348 & 1.0000 & 1.0000 & 1.1062 & 1.0626 \\
\hline & \multirow{4}{*}{0.15} & $\hat{\mu}_{\mathrm{pa}}$ & 1.4042 & 1.1689 & 1.1315 & 1.0511 & 1.0492 \\
\hline & & $\hat{\mu}_{\mathrm{BR} 1}$ & 1.3947 & 1.3222 & 1.1511 & 1.0566 & 1.0340 \\
\hline & & $\hat{\mu}_{\mathrm{BR} 2}$ & 1.0000 & 0.9786 & 1.1074 & 1.0351 & 1.0288 \\
\hline & & $\hat{\mu}_{\mathrm{pa}}$ & 1.0745 & 0.9828 & 1.0266 & 1.0216 & 0.9543 \\
\hline \multirow{5}{*}{$80 \%$} & \multirow[t]{2}{*}{0} & $\hat{\mu}_{\mathrm{BR} 1}$ & 1.0596 & 0.9833 & 1.0044 & 1.0200 & 0.9249 \\
\hline & & $\hat{\mu}_{\mathrm{BR} 2}$ & 0.9718 & 1.0000 & 1.0452 & 1.0502 & 0.9763 \\
\hline & \multirow{3}{*}{0.15} & $\hat{\mu}_{\mathrm{pa}}$ & 1.0676 & 0.9888 & 1.0000 & 1.0044 & 1.0408 \\
\hline & & $\hat{\mu}_{\mathrm{BR} 1}$ & 1.0776 & 1.0066 & 1.0000 & 0.9860 & 1.0000 \\
\hline & & $\hat{\mu}_{\mathrm{BR} 2}$ & 1.0503 & 1.0225 & 1.1019 & 0.9920 & 1.0493 \\
\hline
\end{tabular}

Note: SSE - sampling standard error of the estimator [ = standard deviation of the 500 estimated domain means]; ESE - estimated standard error of the estimator [ = mean of the 500 estimated standard errors of the bootstraps]. $\delta$ - difference between the mean of the $y$-values of the jumpers and the mean of the $y$-values of the rest of the firms in the subdomain, in the finite population; $p_{\text {jump }}$ - percent of jumpers among small firms in the finite population. 
Table 7. Error rate $(E R, \%)$ of $\hat{\mu}_{p a}, \hat{\mu}_{B R 1}$ and $\hat{\mu}_{B R 2}$ under a $20 \%$ and an $80 \%$ response rate.

\begin{tabular}{|c|c|c|c|c|c|c|c|}
\hline \multirow[b]{2}{*}{ Response rate } & \multirow[b]{2}{*}{$\delta$} & \multirow[b]{2}{*}{ Estimator } & \multicolumn{5}{|c|}{$p_{\text {jump }}, \%$} \\
\hline & & & 1 & 2 & 5 & 7 & 10 \\
\hline \multirow{7}{*}{$20 \%$} & \multirow{3}{*}{0} & $\hat{\mu}_{\mathrm{pa}}$ & 12.2 & 9.2 & 6.6 & 11.0 & 7.4 \\
\hline & & $\hat{\mu}_{\mathrm{BR} 1}$ & 12.6 & 10.4 & 10.0 & 11.4 & 10.0 \\
\hline & & $\hat{\mu}_{\mathrm{BR} 2}$ & 21.2 & 23.0 & 7.0 & 8.2 & 6.6 \\
\hline & \multirow{4}{*}{0.15} & $\hat{\mu}_{\mathrm{pa}}$ & 32.0 & 15.4 & 8.0 & 5.8 & 9.2 \\
\hline & & $\hat{\mu}_{\mathrm{BR} 1}$ & 48.0 & 46.4 & 47.6 & 58.0 & 69.0 \\
\hline & & $\hat{\mu}_{\mathrm{BR} 2}$ & 81.6 & 79.0 & 49.0 & 31.8 & 16.2 \\
\hline & & $\hat{\mu}_{\mathrm{pa}}$ & 6.6 & 4.4 & 6.4 & 6.8 & 3.8 \\
\hline \multirow{5}{*}{$80 \%$} & \multirow[t]{2}{*}{0} & $\hat{\mu}_{\mathrm{BR} 1}$ & 7.4 & 4.8 & 5.8 & 7.6 & 3.4 \\
\hline & & $\hat{\mu}_{\mathrm{BR} 2}$ & 37.4 & 22.0 & 7.4 & 11.4 & 19.2 \\
\hline & \multirow{3}{*}{0.15} & $\hat{\mu}_{\mathrm{pa}}$ & 13.0 & 6.0 & 6.2 & 6.8 & 5.6 \\
\hline & & $\hat{\mu}_{\mathrm{BR} 1}$ & 76.4 & 94.4 & 99.2 & 100.0 & 100.0 \\
\hline & & $\hat{\mu}_{\mathrm{BR} 2}$ & 96.4 & 93.2 & 45.6 & 15.2 & 5.6 \\
\hline
\end{tabular}

Note: ER - error rate of the CIs constructed from the percentiles of the bootstrap distribution. $\delta$ - difference between the mean of the $y$-values of the jumpers and the mean of the $y$-values of the rest of the firms in the subdomain, in the finite population; $p_{\text {jump }}$ - percent of jumpers among small firms in the finite population.

\section{Discussion}

In this article, we focused on challenges in estimating the means of domains (defined by the level of firm size) from a business survey in the presence of misclassified frame records (jumpers) and a low response rate. We studied the impact of jumpers on the performance of the weighted ratio estimator with propensity-adjusted weights with and without smoothing of the weights. Our findings and conclusions are based on the results of an extensive simulation study with numerous scenarios of practical relevance.

Our results demonstrate satisfactory performance of the weighted ratio estimator with propensity-adjusted weights for all scenarios under a $20 \%$ response rate, in which the percent of jumpers among small firms in the finite population was greater than or equal to $7 \%$, and all scenarios under an $80 \%$ response rate. Antal-Tille's variance estimator was very close to the sampling variance in these cases and the error rates of the corresponding CIs were satisfactory. For smaller percentages of jumpers and a low response rate, we observed underestimation of variances of the propensity-adjusted domain mean estimator and higher than nominal error rates.

The underestimated variances can, at least partially, be explained by highly variable weights in domains with a very small number of jumpers. Similar findings have been observed in a different context (Li et al. 2011; Landsman and Graubard 2013). Small-sized population domains of large and/or very large firms, typically seen in populations of businesses, combined with a low response rate, may imply a very small number of respondents in the sample domain that can be used for the estimation. In such cases, the asymptotic properties of the weighted ratio estimator may not apply, resulting in a distorted sampling distribution and high error rates for CI coverage. As expected, the results improve considerably under a higher response rate.

The validity of the two smoothed weighted estimators relies on the assumption of conditional independence between the variable of interest and the nonresponse-adjusted 
weights (before smoothing) given the variables that define a subdomain (e.g., firm size collected at the time of survey, geographic region, and business sector). Adapting additional model-assisted methods of improving survey-weighted estimates in the presence of highly variable weights (Chen et al. 2017) to business surveys with misclassified records and exploring their properties empirically is an important direction for future research.

It is important to note that the sampling design in the OLIP and the simulated data, like many other business surveys with voluntary response, can be considered to be a special case of a two-phase sampling design, where a stratified random sample of firms is selected in the first phase and a sample of respondents is self-selected in the second phase. The proposed bootstrap variance estimator does not fully account for the randomness in the second phase, because the response indicator is assumed to be a fixed variable. In general, if the sampling fractions are non-negligible in the first phase (as is the case in the OLIP and the simulated data), both variance components should be accounted for in the variance estimator to avoid variance underestimation (Kim et al. 2006; Beaumont et al. 2015). To address this problem, Kim and $\mathrm{Yu}$ (2011) proposed a multiplicative adjustment to the second phase weights and implemented it for the jackknife variance estimator. However, the jackknife variance estimator is not appropriate for our data due to jumpers and literature on this topic in the framework of bootstrap variance estimator appears to be sparse. At the same time, the results of our simulation study demonstrated a very close correspondence of the estimated standard error (ESE) and sampling standard error (SSE) for the propensity-adjusted estimator in the scenario without jumpers, under both response rates. This implies that the missed variance component might be of a small order in the application that we studied. Still, development and implementation of an improved variance estimator in the bootstrap setting is an important direction for future work.

Given the complexity of the jumpers problem, it is instructive to compare among several estimates rather than sticking to a standard approach. We hope that the analysis of the OLIP data serves as a useful demonstration of this strategy. Although we find it encouraging to see that the various estimates produced fairly similar results for some variables of interest in the application that we studied, we acknowledge that it may not be the case in other applications, since the validity of these estimators relies on strong assumptions that were not verified as part of the current analysis. If an analyst observes substantial discrepancies between the estimates, we would encourage them to explore this problem further and try to understand the reason for it (e.g., outliers, informativeness of the weights), which might help to decide which of the estimates would be the most reliable in a given situation. We would also like to emphasize that our simulation results imply that a small number of jumpers combined with a low response rate is the most difficult scenario for making a valid statistical inference from a business survey. If a practitioner suspects that this is going to be the case in their data, the estimator with nonresponse-adjusted weights (without smoothing) would probably be the safest choice, based on our findings.

\section{References}

Antal, E. and Y. Tillé. 2014. "A New Resampling Method for Sampling Designs Without

Replacement: the Doubled Half Bootstrap." Computational Statistics 29: 1345-1363.

DOI: https://doi.org/10.1007/s00180-014-0495-0. 
Beaumont, J.-F. 2008. “A New Approach to Weighting and Inference in Sample Surveys." Biometrika 95: 539-553. DOI: http:s//doi.org/10.1093/biomet/asn028.

Beaumont, J.-F., A. Beliveau, and D. Haziza. 2015. "Clarifying Some Aspects of Variance Estimation in Two-Phase Sampling." Journal of Survey Statistics and Methodology 3: 524-542. DOI: https://doi.org/10.1093/jssam/smv022.

Beaumont, J.-F., and Z. Patak. 2012. "On the Generalized Bootstrap for Sample Surveys with Special Attention to Poisson Sampling." International Statistical Review 80: 127-148. DOI: https://dx.doi.org/10.1111/j.1751-5823.2011.00166.x.

Beaumont, J.-F., and L.-P. Rivest. 2009. "Dealing with Outliers in Survey Data." In Handbook of Statistics, vol. 29A, Sample Surveys: Design, Methods and Applications, edited by D. Pfeffermann and C.R. Rao, 247-279. Amsterdam: Elsevier. DOI: https:// doi.org/10.1016/S0169-7161(08)00011-4.

Chen, Q., M.R. Elliott, D. Haziza, Y. Yang, M. Ghosh, R.J.A. Little, J. Sedransk, M. Thompson. 2017. "Approaches to improving survey-weighted estimates". Statistical Science 32(2): 227-248. DOI: https://doi.org/10.1214/17-STS609.

Cook, S., P. LeBaron, L. Flicker, and T.S. Flanigan. 2009. "Applying Incentives to Establishment Surveys: A Review of the Literature.” In Proceedings of the Section on Survey Research Methods: American Statistical Association, May 14-17, 2009. 5639-5647. Available at: http://www.asasrms.org/Proceedings/y2009/Files/400022. pdf (accessed March 2019).

Favre-Martinoz, C., D. Haziza, and J.-F. Beaumont. 2015. "A Method of Determining the Winsorization Threshold, With an Application to Domain Estimation." Survey Methodology 41: 57-77.

Haziza, D., and E. Lesage. 2016. "A Discussion of Weighting Procedures for Unit Nonresponse.” Journal of Official Statistics 32: 129-145. DOI: https://doi.org/10.1515/ JOS-2016-0006.

Institute for Work and Health. 2011. "Benchmarking Organizational Leading Indicators for the Prevention and Management of Injuries and Illnesses: Final Report." Available at: https://www.iwh.on.ca/sites/iwh/files/iwh/reports/iwh_report_benchmarking_organizational_leading_indicators_2011.pdf (accessed March 2019).

Institute for Work and Health. 2013. "Measures in the Ontario Leading Indicators Project (OLIP) Survey.” Available at: https://www.iwh.on.ca/sites/iwh/files/iwh/reports/iwh_ project_olip_about_the_measures_august_2013.pdf (accessed March 2019).

Kim., J.K., and J.J. Kim. 2007. "Nonresponse Weighting Adjustment Using Estimated Response Probability." The Canadian Journal of Statistics 35: 501-514. DOI: https:// doi.org/10.1002/cjs.5550350403.

Kim, J.K., A. Navarro, and W.A. Fuller. 2006. "Replication Variance Estimation for TwoPhase Stratified Sampling." Journal of the American Statistical Association 101: $312-320$.

Kim, J.K., and C.L. Yu. 2011. "Replication Variance Estimation Under Two-phase Sampling." Survey Methodology 37: 67-74.

Korn, E.L., and B.I. Graubard. 1999. Analysis of Health Surveys. New York: Wiley. DOI: https://doi.org/10.1002/9781118032619.fmatter.

Lachin, J.M. 2011. Biostatistical Methods: The Assessment of Relative Risks. 2nd edition, Hoboken, NJ: Wiley. DOI: https://doi.org/10.1002/sim.1167. 
Landsman, V., and B.I. Graubard. 2013. "Efficient Analysis of Case-control Studies With Sample Weights." Statistics in Medicine 32: 347-360. DOI: https://doi.org/10.1002/ sim.5530.

Lee, H. 1995. "Outliers in Business Surveys.” In Business Survey Methods, edited by B.G. Cox, D.A. Binder, B.N. Chinnappa, A. Christianson, M.J. Colledge and P.S. Kott, 503-526. New York: Wiley. DOI: https://doi.org/10.1002/9781118150504.ch26.

Li, Y., B.I. Graubard, and R. DiGaetano. 2011. "Weighting Methods for Population-based Case-control Studies With Complex Sampling." JRSS(C) 60: 165-185. DOI: https:// doi.org/10.1111/j.1467-9876.2010.00731.x.

Lohr, S.L., M.K. Riddles, and D. Morganstein. 2016. “Tests for Evaluating Nonresponse Bias in Surveys." Survey Methodology 42: 195-218.

Little, R.J., and S. Vartivarian. 2003. "On Weighting the Rates in Non-response Weights." Statistics in Medicine 22: 1589-1599. DOI: https://doi.org/10.1002/sim.1513.

MacNeil, D., and S. Pursey. 2002. "Dealing With Industry Misclassifications in the Unified Enterprise Survey." In Proceedings of the Survey Methods Section: SSC Annual Meeting, May 2002: 51-56.

Mashreghi, Z., D. Haziza, and C. Léger. 2016. "A Survey of Bootstrap Methods in Finite Population Sampling." Statistics Surveys 10: 1-52. DOI: https://doi.org/10.1214/16SS113.

Nordlöf, H., K. Wijk, and K.-E. Westergren. 2015. "Perceptions of Work Environment Priorities: Are there any Differences by Company Size? - An Ecological Study." Work 52: 697-706. DOI: https://doi.org/10.3233/WOR-152123.

Pfeffermann, D., and M. Sverchkov. 1999. "Parametric and Semi-parametric Estimation of Regression Models Fitted to Survey Data.” Sankhya B 61: 166-186.

Rao, J.N.K. 1966. "Alternative Estimators in PPS Sampling for Multiple Characteristics." Sankhya: The Indian Journal of Statistics 28: 47-60.

Rao, J.N.K. and C.F.J. Wu. 1988. "Resampling Inference With Complex Survey Data." Journal of the American Statistical Association 83: 231-241. DOI: https://doi.org/10. 1080/01621459.1988.10478591.

Rao, J.N.K., C.F.J. Wu, and K. Yue. 1992. “Some Recent Work on Resampling Methods for Complex Surveys.” Survey Methodology 18: 209-217.

Valliant, R., J.A. Dever, and F. Kreuter. 2013. "Basic Steps in Weighting.” In Practical Tools for Designing and Weighting Survey Samples, edited by R. Valliant, J.A. Dever, and F. Kreuter, 307-348. New York: Springer. DOI: https://doi.org/10.1007/978-3319-93632-1_13.

U.S. Department of Labor; Bureau of Labor Statistics; Office of Survey Methods Research. Household and establishment survey response rates. Chart 2. Establishment surveys: overall unit response rates. Washington DC. Available at: https://www.bls.gov/ osmr/response-rates/home.htm (accessed October 2019).

Received March 2019

Revised October 2019

Accepted May 2021 